\title{
Long-term synaptic changes in two input pathways into the lateral nucleus of the amygdala underlie fear extinction
}

\author{
Junchol Park and June-Seek Choi ${ }^{1}$ \\ Department of Psychology, Korea University, Seoul 136701, South Korea
}

\begin{abstract}
Plasticity in two input pathways into the lateral nucleus of the amygdala (LA), the medial prefrontal cortex (mPFC) and the sensory thalamus, have been suggested to underlie extinction, suppression of a previously acquired conditioned response (CR) following repeated presentations of the conditioned stimulus (CS). However, little is known about the joint dynamics of the relevant synaptic changes within the LA that accompany fear extinction. Employing a novel training procedure, in which stimulation of the medial geniculate nucleus $(\mathrm{MGm})$ of the thalamus served as the CS, we tested necessary and sufficient conditions for extinction in anesthetized rats. Repeatedly applying the brain-stimulation CS was neither sufficient to produce activation of the MPFC nor behavioral extinction when the animal was under anesthesia. Only when the CS was combined with contingent stimulation of the infralimbic cortex (IL) of the MPFC was the CR markedly reduced, emulating extinction. To elucidate the nature of synaptic alterations linking the extinction procedure with CR suppression, evoked field potentials to IL and MGm stimulations were recorded in the LA. The results showed that paired stimulations of the IL and MGm significantly enhanced the neural response at the IL-LA synapses and reversed conditioning-induced synaptic potentiation at the MGm-LA synapses. Taken together, our results provide strong evidence that dual plasticity within the LA underlies suppression of conditioned fear response following extinction.
\end{abstract}

The lateral nucleus of the amygdala (LA) has been implicated as a key site for learning-related plasticity following Pavlovian fear conditioning, in which a neutral conditioned stimulus (CS) is associated with a noxious unconditioned stimulus (US) (Davis et al. 1994; Fanselow and LeDoux 1999; Maren and Quirk 2004). Evidence comes from multiple levels. Fear conditioning induces long-term enhancement in reactivity of LA neurons (Rogan and LeDoux 1995; McKernan and Shinnick-Gallagher 1997; Rogan et al. 1997; Kwon and Choi 2009). Protein synthesis in the LA is required for consolidation of Pavlovian fear memory (Schafe and LeDoux 2000; Schafe et al. 2000, 2001; Rodrigues et al. 2004). In addition, synaptic alterations within the LA have been suggested to underlie fear extinction, which is the decline of a previously acquired conditioned response (CR) due to repeated presentations of the CS without the US (for review, see Myers and Davis 2007; Quirk and Mueller 2008). Inactivation of the $N$-methyl-D-aspartic acid (NMDA) receptors and mitogen-activated protein kinase/ extracellular-signal regulated kinase (MAPK/ERK) within the LA impaired extinction of fear CRs, supporting a role for neuronal plasticity in the LA during the acquisition of extinction memory (Falls et al. 1992; Lee et al. 2001; Herry et al. 2006; Sotres-Bayon et al. 2007). In addition, unit-recording studies in the LA have shown that enhanced neuronal activity to the tone CS following conditioning was significantly diminished after extinction (Quirk et al. 1995), while a different subset of neurons showed persistent firings to the CS even after extinction (Repa et al. 2001).

Among the input projections to the LA, two are particularly relevant for the extinction of fear memory: the medial division of the medial geniculate nucleus (MGm) of the auditory thalamus and the medial prefrontal cortex (mPFC). The MGm sends direct

\footnotetext{
'Corresponding author.
}

E-mail j-schoi@korea.ac.kr; fax 82-2-32902662.

Article is online at http://www.learnmem.org/cgi/doi/10.1101//m.1482910. projections and transmits auditory information to the LA (LeDoux et al. 1990a,b, 1991). The thalamo-amygdala synapses, therefore, have been a prime candidate for the site of conditioning-related plasticity (Blair et al. 2001). Recently, however, extinction-related synaptic plasticity also has been reported in these synapses. Synaptic potentiation in the thalamic input pathway, induced by fear conditioning, was reversed following repetitive CS exposure. In addition, extinction training reversed conditioninginduced surface expression of AMPA receptors within LA synapses (Mao et al. 2006; Kim et al. 2007).

The $\mathrm{mPFC}$ has been implicated as a key site for fear extinction. Lesion or inactivation of the mPFC slowed or impaired extinction learning (Quirk et al. 2000; Sierra-Mercado et al. 2006), and stimulation of the infralimbic region (IL) of the mPFC paired with the tone CS facilitated fear extinction (Milad et al. 2004). Consistent with these findings, extinction training enhanced neural plasticity in the mPFC and its afferents (Herry and Garcia 2002; Milad and Quirk 2002). In addition to the intracortical changes, activation of the mPFC has been suggested to contribute to extinction-related synaptic changes within the amygdala (Rosenkranz et al. 2003; Likhtik et al. 2008). Moreover, modified connections between the mPFC and other areas in the fear memory circuit, e.g., inhibitory interneurons in the amygdala (Bauer and LeDoux 2004), have been suggested to underlie suppression of fear response after extinction.

Based on the known contributions of the auditory thalamic and prefrontal input pathways onto the LA to fear memory formation and alteration, we hypothesized that synaptic changes within the LA underlie behavioral extinction. We tested whether inducing synaptic alterations in the two input pathways into the LA would lead to reduction of the fear CR. To address this, a novel extinction training protocol was designed, in which the conventional extinction training was replaced with electrical stimulation of the MGm and the IL (Fig. 1). In addition, the extinction training 
was given while the rats were under anesthesia. We expected that methodologically combining the reduced circuit and general anesthesia would provide unique advantages over conventional extinction procedures in linking cellular changes to a memory trace. First, with the moving animal, changes in a behavioral response cannot be solely attributable to the synaptic plasticity because behavior could interfere with or even inversely induce the cellular change. In the current design, however, an extinction-like process was induced in anesthetized animals using only the local brain stimulations that do not involve any external movement. Consequently, a causal relationship between cellular plasticity and a subsequent behavioral change can be firmly defined. Second, general anesthesia can effectively depress various cortical areas (e.g., auditory cortex), some of which make direct connections with the amygdala. Combined with local activation of selected cortical regions, anesthesia is a way of controlling nonessential neural components. In addition, evoked field potentials (EFPs), often employed to probe synaptic plasticity, are reliably measured while the animal is under anesthesia. Therefore, anesthesia creates a drastic context shift between two critical sessions: behavioral measurement, which is performed while conscious and ambulatory; and neurophysiological measurement, which is executed while anesthetized. By applying brain stimulation as the CS to anesthetized rats during extinction, the physiological state remains constant during the two critical sessions (anesthetized during both extinction training and EFP measurement).

\section{Results}

\section{Experiments 1 and 2: Effects of extinction training under anesthesia}

Although an association between a neutral CS and an aversive US can be established in an anesthetized animal, as evidenced by behavioral (Weinberger et al. 1984; Gold et al. 1985) and neural data (Rosenkranz and Grace 2002b), whether extinction could occur in the same condition has not been tested. We first trained conscious, behaving rats using the auditory CS. During the extinction training, rats in the Anesthetized-Ext group were anesthetized and exposed to 15 CS-only presentations. In the subsequent extinction retention test, these rats showed high levels of freezing to the tone CS, equivalent to that of the Anesthetized group, which did not receive extinction training (no group effect, $F_{(1,10)}=$ $1.51, P>0.2$; no group $\times$ trial effect, $\left.F_{(4,40)}=1.63, P>0.1\right)$, indicating that the extinction training was ineffective under anesthesia (Fig. 2A).

Lack of extinction under anesthesia might be due to the lack of activation in the auditory input to the amygdala. To rule out this possibility, the auditory CS was replaced with electrical stimulation of the MGm in experiment 2. Otherwise, experimental protocol was identical to ex- periment 1: Rats were fear conditioned using the brain-stimulation CS while they were conscious and received extinction training under anesthesia. During the extinction training, repeated brain stimulation $(\times 15)$ did not evoke any visible movement or activity in anesthetized animals. When tested in the retention session, the rats in the MGm-stim group froze to the test CS as much as the rats in the Anesthetized group (no group effect, $F_{(1,14)}=0.314, P>0.5$; no group $\times$ trial interaction, $\left.F_{(4,56)}=0.62, P>0.5\right)$, indicating that the extinction training was ineffective under anesthesia even with the brain-stimulation CS (Fig. 2B).

\section{Experiment 3: Effects of extinction training under anesthesia on neuronal activities in the $\mathrm{mPFC}$}

Given the lack of behavioral suppression, despite the extinction training under anesthesia, to neither the auditory nor the brainstimulation CS, we reasoned that cortical suppression induced by anesthesia impaired prefrontal activity during the extinction training and in turn blocked extinction learning. We next investigated whether depressed mPFC activity during extinction
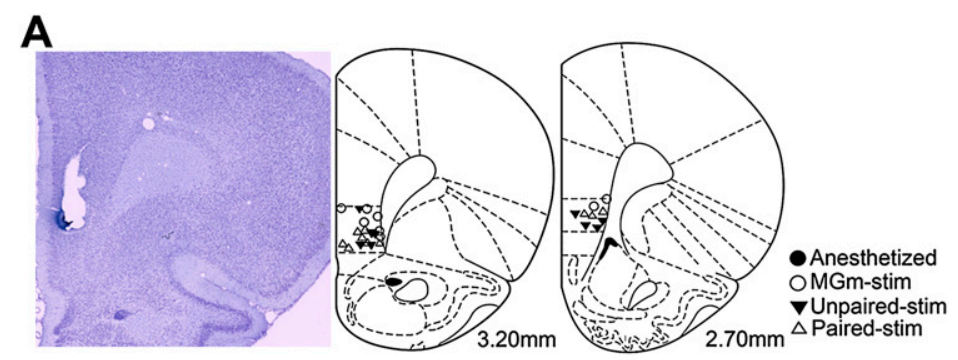

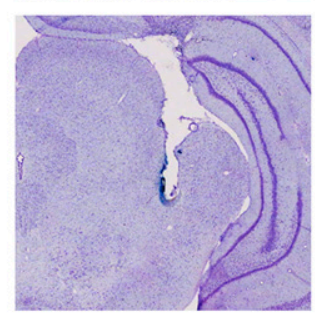

B
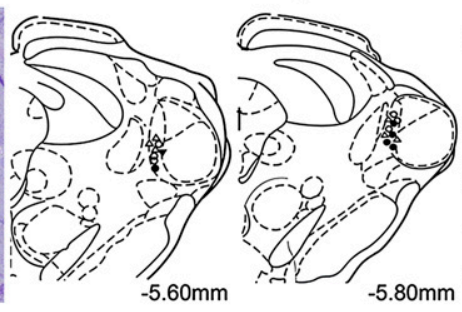

D
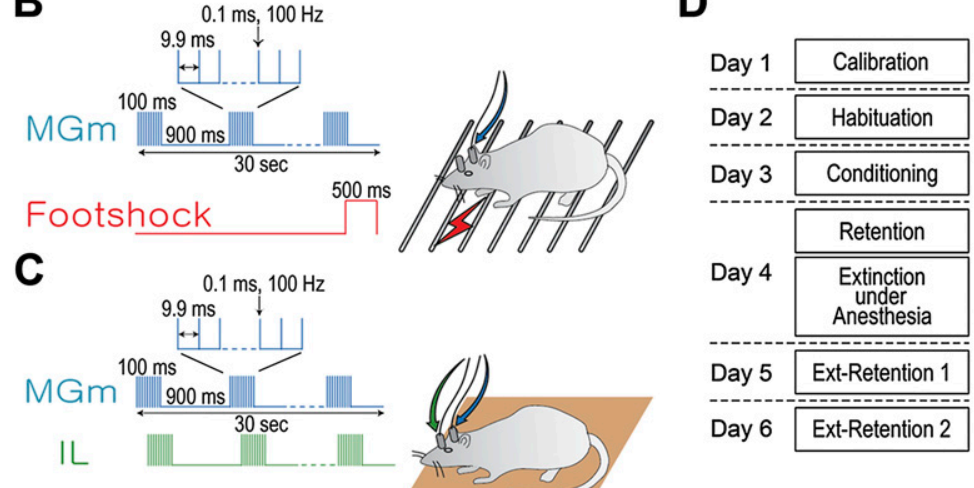

Figure 1. Reconstruction of electrode placement and experimental procedure. $(A)$ The photomicrographs show cresyl-violet-stained coronal sections of the IL (top left) and MGm (bottom left). A series of illustrated coronal sections are presented through the rostro-caudal extent of the IL (top right) and $\mathrm{MGm}$ (bottom right), showing the location of stimulation sites (modified from Paxinos and Watson 1998 and reprinted with permission from Elsevier (c1998). The numbers at the bottom represent distance from bregma. (B) For fear conditioning, MGm stimulation (30 trains of 10, 100- $\mu$ sec square pulses at $100 \mathrm{~Hz}$ with 1 -sec intertrain intervals) was used as the CS and 500-msec footshock as the US. A total of five CS-US pairings were delivered to a conscious, behaving rat. (C) During the extinction training, the MGm-stimulation CS was delivered alone or in combination with the IL stimulation (same stimulation parameters as the MGm stimulation) to an anesthetized rat. Each rat received 15 trials. (D) Experimental procedure for experiments 2 and 4. 

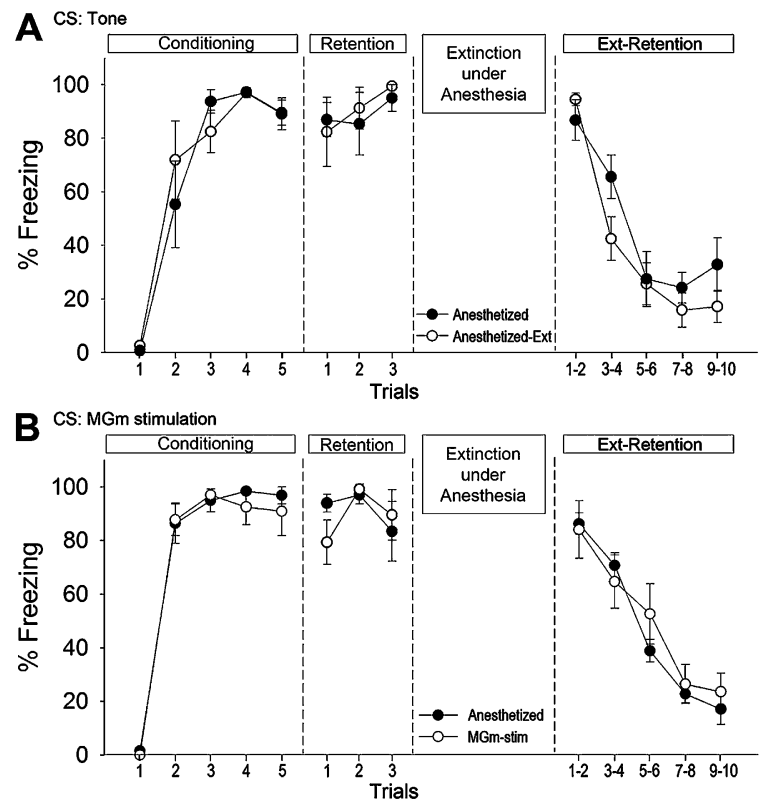

Figure 2. Conditioning and extinction under anesthesia with external and brain-stimulation CSs. Data points were averaged across two trial blocks during the extinction-retention session (10 trials). ( $A$ ) Rats acquired normal fear CRs to the auditory CS as evidenced by a high level of freezing during the conditioning and retention test. Extinction procedure was ineffective when administered under anesthesia as the level of freezing during the extinction retention test was indiscriminative between the Anesthetized-Ext and Anesthetized groups. (B) Similarly, normal fear CRs were developed to the MGm-stimulation CS following conditioning, but the extinction training was ineffective when administered under anesthesia.

training under anesthesia blocked the effect of extinction learning. Extinction requires activation of the mPFC neurons as evidenced by a series of data showing immediate early gene expression in the mPFC (Herry and Mons 2004; Santini et al. 2004), potentiation of intrinsic excitability of the IL neurons (Santini et al. 2008), as well as extinction-related synaptic potentiation in the thalamic input pathway to the mPFC (Herry and Garcia 2002). Using c-Fos immunohistochemistry, we tested whether extinction training under anesthesia induced functional activation in the mPFC. Rats were fear conditioned while conscious using MGm stimulation as the CS and received extinction training either in an awake or anesthetized state. After completion of the extinction training, the number of c-Fos immunoreactive neurons, an index of functional activation (Kaczmarek and Robertson 2002), was measured in the mPFC subregions.

In the conditioning session, there was no significant difference in the freezing level among all the groups. All groups displayed equivalent freezing levels to the final CS $\left(F_{(2,11)}=1.26\right.$, $P>0.3$ ) (Fig. 3C). At the presentation of the test CS, the No-Ext group, which did not receive extinction training, showed an elevated freezing level $(84.97 \pm 6.37 \%)$, whereas the Ext group, which received 15 CS-alone trials without anesthesia, showed a significantly reduced freezing level $(10.55 \pm 4.74 \%)$ that was comparable to those of Stimulation-Only and Naïve groups (4.77 \pm $1.01 \%$ and $2.09 \pm 0.29 \%$, respectively) (Fig. 3D). One-way ANOVA revealed a significant main effect of group $\left(F_{(3,14)}=95.22, P<\right.$ $0.001)$, with Bonferroni's post hoc comparison confirming that the No-Ext group froze significantly more than all the other groups ( $P$-values $<0.01$ ).

Repeated CS presentations yielded significantly different Fos expression levels in the mPFC depending on whether the rats were anesthetized or conscious during the extinction training (Fig. $3 \mathrm{~A}, \mathrm{~F})$. One-way ANOVAs revealed significant main effects of group for the number of Fos-IR neurons in both IL $\left(F_{(4,18)}=10.44, P<\right.$ $0.001)$ and prelimbic $(\mathrm{PL})\left(F_{(4,18)}=14.84, P<0.001\right)$ subregions of the mPFC, but not in the primary motor cortex $(\mathrm{M} 1)\left(F_{(4,18)}=1.55\right.$, $P>0.2$ ). Bonferroni's post hoc comparisons showed that Fos expression of the Ext group was significantly greater than the other groups in both IL $(22.57 \%)$ and PL $(26.96 \%)(P$-values $<0.05)$, suggesting that the extinction training elicited Fos expression in the mPFC, as reported in previous studies. In contrast to the Ext group, rats in the No-Ext (IL: $14.05 \%$, PL: $11.06 \%$ ), AnesthetizedExt (IL: $8.82 \%$, PL: $12.60 \%)$ groups, as well as Stimulation-Only (IL: 10.89\%, PL: $11.28 \%$ ) and Naïve (IL: 9.09\%, PL: $8.80 \%)$ control groups, exhibited low Fos expression levels in both IL and PL ( $P$-values $>0.6)$. This indicates that the mPFC was not functionally activated despite the same amount of extinction training, when the animal was anesthetized. Together, these findings imply that the lack of reduction in CR following the extinction procedure might be attributable to the suppressed activity of mPFC neurons during the anesthesia.

\section{Experiment 4: Effects of IL stimulation on extinction training under anesthesia}

Repetitive presentations of the CS under anesthesia failed to produce behavioral extinction, perhaps due to the lack of functional activation in the $\mathrm{mPFC}$, as suggested by previous studies (Santini et al. 2004, 2008). We hypothesized, therefore, that stimulating IL neurons during extinction training might rescue the extinction memory, which was impaired due to suppressed cortical function under anesthesia. As a way of artificially inflating the activity, the IL was given electrical stimulation, either paired or unpaired with the MGm stimulation CS, during the extinction training under anesthesia.

Rats were fear conditioned using MGm stimulation as the CS. All groups showed equivalent freezing levels to the CS across the conditioning and retention sessions ( $P$-values $>0.5$ ) (Fig. 4A). The data from the Anesthetized and MGm-stim groups in experiment 2 were also taken into the statistical comparison. Most notably, when tested $24 \mathrm{~h}$ after extinction training under anesthesia, rats in the Paired-stim group, which received paired stimulations $(\times 15)$ of the MGm and IL, displayed markedly reduced freezing levels (Fig. 4B). A repeated-measures ANOVA revealed a significant main effect of group $\left(F_{(3,31)}=13.68, P<0.001\right)$, trial block $\left(F_{(4,124)}=\right.$ 68.87, $P<0.001)$, and the interaction $\left(F_{(12,124)}=2.47, P<0.01\right)$. A post-hoc analysis (Bonferroni's procedure) revealed that the Paired-stim group froze significantly less than all the other groups in trial blocks 1 and 2 ( $P$-values $<0.05)$. However, the freezing level was not reduced in the Unpaired-stim group despite the same number of MGm and IL stimulations. The Unpaired-stim group displayed freezing levels comparable to those of the Anesthetized and MGm-stim groups in all trial blocks ( $P$-values $>0.5)$. In the second extinction retention session, administered $48 \mathrm{~h}$ after the extinction training, rats in the Paired-stim group showed lower spontaneous recovery of fear $(22.49 \pm 5.27 \%)$ compared with the MGm-stim (46.87 $\pm 7.27 \%$ ) and Unpaired-stim (54.97 $\pm 5.90 \%$ ) groups (Fig. 4B). One-way ANOVA revealed a significant main effect of group $\left(F_{(3,31)}=5.76, P<0.01\right)$. A post hoc analysis (Bonferroni's procedure) confirmed that the rats in the Paired-stim group showed significantly lower spontaneous recovery of fear than rats in the Unpaired-stim or MGm-stim group ( $P$-values $<0.05$ ).

In sum, extinction training under anesthesia required paired stimulations of IL and MGm input to the LA. MGm stimulation alone or MGm stimulation with asynchronous IL stimulation failed to induce any change in behavior when subsequently tested awake. 


\section{Experiment 5: Long-term synaptic changes in the MGm-LA and IL-LA pathways}

To elucidate the nature of synaptic alterations within the LA linking cellular plasticity in the LA with CR reduction, we next performed a series of EFP recordings to examine the synaptic response of the LA neurons (Fig. 5, 6A,B). Rats were fear conditioned with the MGm-stimulation CS and underwent the paired stimulation procedure used in experiment 4 . Synaptic plasticity of the MGmLA and IL-LA pathways were monitored throughout the habituation (Baseline EFP), conditioning (Post-Con EFP), and extinction training with the paired stimulation of the MGm and IL (Post-Ext EFP). The EFP recordings were performed $14 \mathrm{~h}$ after every behavioral session.

The mean change in the EFP amplitude and the latency to the first negative peak were analyzed for all recording sessions (Table 1). For MGm stimulationinduced EFPs, repeated-measures ANOVA revealed that the mean amplitudes were different between pre- and post-extinction sessions $\left(F_{(2,18)}=22.22, P<0.01\right)$. There was no significant effect of stimulation group $\left(F_{(1,9)}=2.60, P>0.05\right)$. However, the interaction was significant $\left(F_{(2,18)}=\right.$ 4.52, $P<0.05)$. To further analyze the nature of the interaction, the EFP amplitudes were compared among sessions for each group. For both groups, the mean EFP magnitude in the MGm-LA pathway increased significantly after fear conditioning, compared to the baseline potential measured before conditioning (Paired-stim: $128.28 \pm 3.79 \%$, Unpairedstim: $124.37 \pm 6.05 \%$ ) (Fig. 6D,E). Bonferroni's post-hoc analysis confirmed that the mean EFP amplitude of the PostCon session was significantly greater than that of the baseline for both groups $(P$-values $<0.05)($ Fig. 6D,E). This result is consistent with previous studies showing enhanced synaptic efficacy of the MGm-LA pathway following fear conditioning. However, repeated IL stimulations given paired with the CS significantly decreased the magnitude of the EFPs (Paired-stim: $103.07 \pm 4.10 \%$ ). The Post-Ext EFP amplitude was significantly lower than that of the Post-Con session $(P<0.05)$, but equivalent to that of the baseline $(P>0.5)$. The mean EFP amplitude of the Unpaired-stim group $(121.42 \pm 5.09 \%)$, on the contrary, remained elevated from the baseline $(P<$ 0.05), despite the repeated IL stimulations. The Post-Ext EFP of the Unpairedstim group was significantly greater relative to that of the Paired-stim group $(P<$ 0.05 ). These results indicate that timing of the IL stimulation determines the synaptic plasticity of the MGm-LA pathway.

Behavioral responses were also analyzed in conjunction with the EFP data.

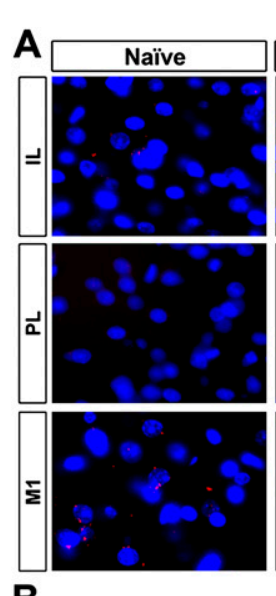

B

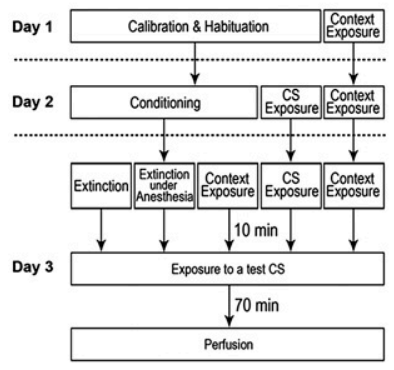

F

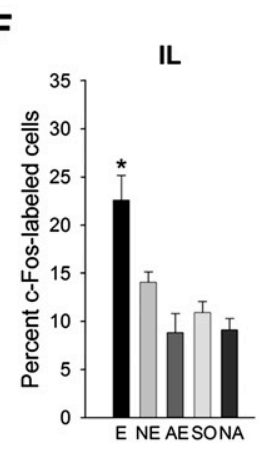

Rats in the Paired-stim group froze markedly less compared to the rats in the Unpaired-stim group when tested $24 \mathrm{~h}$ after the extinction training (Fig. 6C). A repeated-measures ANOVA revealed a significant main effect of group $\left(F_{(1,9)}=8.77, P<0.05\right)$, trial block $\left(F_{(4,36)}=3.77, P<0.05\right)$, and a significant interaction $\left(F_{(4,36)}=2.86, P\right.$ $<0.05)$. Moreover, the Paired-stim group showed a tendency toward less spontaneous recovery of fear responses compared to the Unpaired-stim group when tested on the next day $(P=0.09)$ (Fig. 6C).
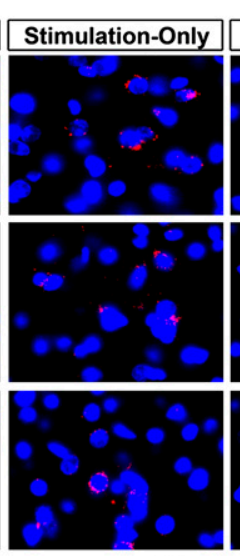

C

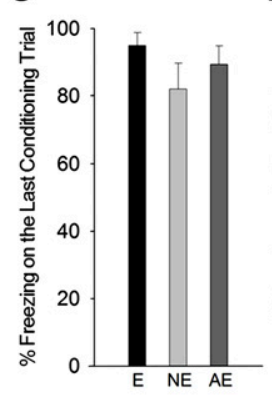

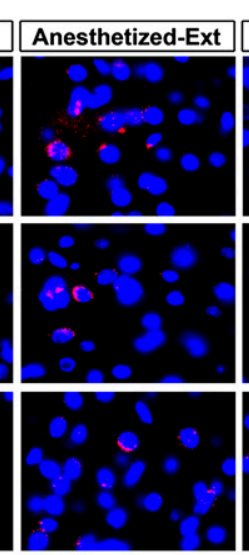

D

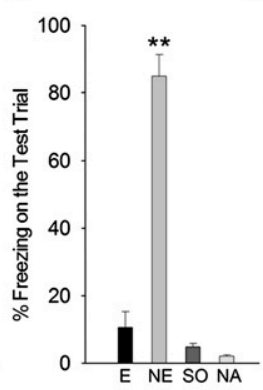

$\mathrm{E}$

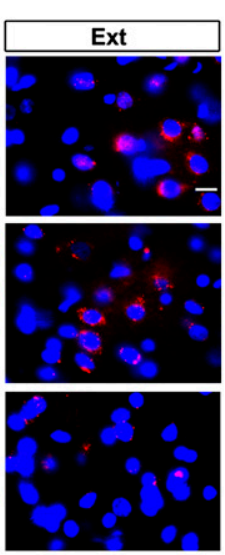

Figure 3. Fos expression in the MPFC after extinction. $(A)$ Photomicrographs of representative samples showing Fos-IR cells in the IL, PL, and M1. Fos expression was detected by CY3 fluorescent dyes (red). Neurons were identified with DAPI staining (blue) in the nucleus. Repeated CS presentations differentially affected Fos expression in the mPFC depending on whether the rats were awake or anesthetized during the extinction procedure. Scale bar $=10 \mu \mathrm{m}$. (B) Experimental procedures for each group in experiment 3. (C) Fear acquisition to the $\mathrm{MGm}$-stimulation CS. All conditioning groups displayed high levels of freezing to the CS on the last acquisition trial. On the next day, the Ext group was presented with 15 CS-only trials in an awake state, whereas the Anesthetized-Ext group received the same amount of CS presentations under anesthesia. (D) Fear response to the test CS. Only the NoExt group showed a high level of freezing to the CS. All other groups, Ext, Stimulation-only, and Naïve groups displayed little freezing. $(E)$ Histological reconstruction of the stimulating electrodes in the MGm mapped onto the matching coronal sections (modified from Paxinos and Watson 1998 and reprinted with permission from Elsevier (C1998). (F) Proportion of Fos-IR neurons in the IL and PL and the control region (M1). Only in the Ext group, which received extinction training while awake, was an elevated level of Fos activation detected in the IL and the PL. No such activation was found in all other groups indicating that extinction may depend on active cortical circuits. Not all cortices are necessary however, as Fos expression was similar for M1 across all groups. (G) Fos-IR neurons were examined in the mPFC (PL and IL) and the primary motor cortex (M1). ${ }^{\star} P<0.05,{ }^{* *} P<0.01$. 
A

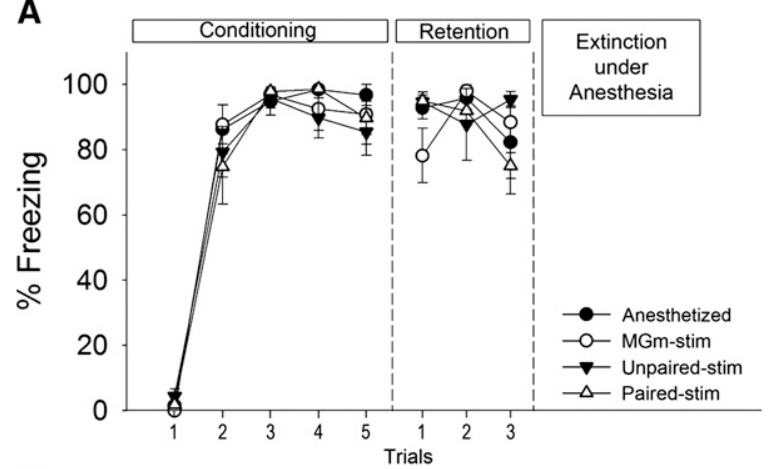

B

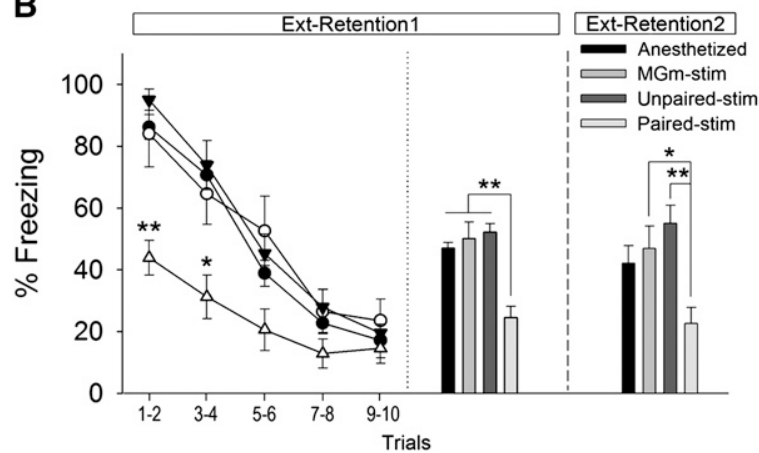

Figure 4. Effect of repetitive $M G m$ and IL stimulations under anesthesia on behavioral extinction. (A) Acquisition of fear CR. In experiment 4, rats were fear conditioned using $\mathrm{MGm}$ stimulation as the CS, and the retention of conditioned fear memory was tested on the following day. There was no group difference in freezing ratio over the conditioning and retention sessions. Anesthetized immediately after the retention test, the Paired-stim group received $15 \mathrm{MGm}$ stimulation paired with IL stimulation, whereas the Unpaired-stim group received explicitly unpaired stimulations. The $\mathrm{MGm}$-stim group received $\mathrm{MGm}$ stimulation only and the Anesthetized group received no such stimulation. (B) Extinction of fear CR. The CR rate of the Paired-stim group was reduced compared to other groups. Unpaired-stim, MGm-stim, and Anesthetized groups continued to display elevated levels of freezing in subsequent extinction retention sessions. ${ }^{*} P<0.05,{ }^{* *} P<0.01$.

Stimulation of the IL also elicited EFPs in the LA, which peaked after $\sim 22-29 \mathrm{msec}$. The shape and peak latency of these potentials were highly reproducible across the rats tested. The mean change in EFP amplitude and the latency to the first negative peak over all recording sessions are shown in Table 1. A repeated-measures ANOVA revealed that the mean EFP amplitudes were different between pre- and post-extinction sessions $\left(F_{(2,18)}=\right.$ $6.71, P<0.01)$. There was no significant effect of stimulation group $\left(F_{(1,9)}=4.13, P>0.05\right)$. However, the interaction was significant $\left(F_{(2,18)}=10.63, P<0.01\right)$. Unlike the MGm-LA pathway, the EFP amplitude of the IL-LA pathway was not altered from the baseline after fear conditioning in both groups (Paired-stim: $102.87 \pm 9.89 \%$, Unpaired-stim: $106.28 \pm 10.79 \%$ ) (Fig. 6F,G). Bonferroni's post-hoc analysis confirmed that the mean EFP amplitude of baseline and Post-Con sessions did not differ $(P$-values $>0.5)$. However, the Paired-stim group showed significantly increased EFP amplitudes $(157.57 \pm 11.42 \%)$ after the repeated pairings of MGm and IL stimulation (Fig. 6F,G) $(P<0.05)$. In contrast, the mean EFP amplitude of the Unpaired-stim group $(96.38 \pm 13.30 \%)$ remained unaltered from the baseline level $(P>$ $0.5)$, despite the same number of MGm and IL stimulations. The Post-Ext EFP of the Paired-stim group was significantly greater than that of the Unpaired-stim group $(P<0.05)$. These data indicate that the high-frequency stimulation applied to the IL alone is not sufficient to induce synaptic potentiation within the IL-LA pathway. Repeated co-activation of the two input structures into the LA, the MGm, and the IL, was necessary to induce LTPlike synaptic change within the IL-LA pathway.

In sum, stimulations of the two input pathways into the LA produced distinctive patterns of synaptic alteration that depended on the temporal arrangement of the stimulations. Specifically, paired stimulation of the MGm and IL yielded reversal of the potentiated MGm-LA response, while significantly enhancing ILLA response. On the contrary, the unpaired stimulation resulted in unaltered synaptic strength in both input pathways. Only in the Paired-stim group was the fear response significantly reduced in the subsequent test session, emulating extinction in awake animals. Taken together, these findings suggest that reorganization of the dual plasticity within the LA contributes to reduction of the previously acquired fear CR.

\section{Discussion}

The current study investigated the nature of synaptic modifications that accompany extinction, especially within IL-LA and MGm-LA pathways. Despite the repeated delivery of the CS, extinction was blocked under anesthesia with either the auditory or the brain-stimulation CS. Using c-Fos immunohistochemistry, we found that the lack of functional activation in the mPFC accompanied the blockade of extinction memory. The subsequent experiment revealed that electrical stimulation of the IL given paired with the CS rescued extinction even under anesthesia. Finally, recorded EFPs in the MGm-LA/IL-LA pathways showed that repeated co-activation of the two amygdalar afferents produced long-lasting modifications of the neuronal responses in the LA and in turn, profoundly reduced freezing when measured in a freely behaving state. Taken together, the dual plasticity within the LA appears to underlie long-term suppression of previously acquired fear memory, and that activation of the IL is a necessary

Table 1. The mean EFP amplitude (percent \pm SEM) and the mean peak latency of the MGm-LA and the IL-LA pathways are shown by group across all recording sessions

\begin{tabular}{|c|c|c|c|c|c|}
\hline \multirow[b]{2}{*}{ Group } & \multicolumn{2}{|c|}{ Amplitude mean change (\%) } & \multicolumn{3}{|c|}{ Mean latency (msec) } \\
\hline & Post-Con & Post-Ext & Base & Post-Con & Post-Ext \\
\hline \multicolumn{6}{|l|}{ MGm-LA } \\
\hline Paired-Stim & $128^{*} \pm 4$ & $103 \pm 4$ & $7.5 \pm 1$ & $7.2 \pm 1$ & $7.2 \pm 1$ \\
\hline Unpaired-Stim & $124^{*} \pm 6$ & $121^{*} \pm 4$ & $6.5 \pm 1$ & $6.5 \pm 1$ & $6.4 \pm 1$ \\
\hline \multicolumn{6}{|l|}{ IL-LA } \\
\hline Paired-Stim & $103 \pm 10$ & $158^{*} \pm 11$ & $28.4 \pm 0.8$ & $27.8 \pm 0.5$ & $27.9 \pm 0.1$ \\
\hline Unpaired-Stim & $106 \pm 11$ & $96 \pm 13$ & $25.9 \pm 0.9$ & $26.0 \pm 1$ & $25.5 \pm 1$ \\
\hline
\end{tabular}

Data showing significantly increased EFP magnitude relative to the baseline level are marked with two asterisks. ${ }^{*} P<0.05$. 

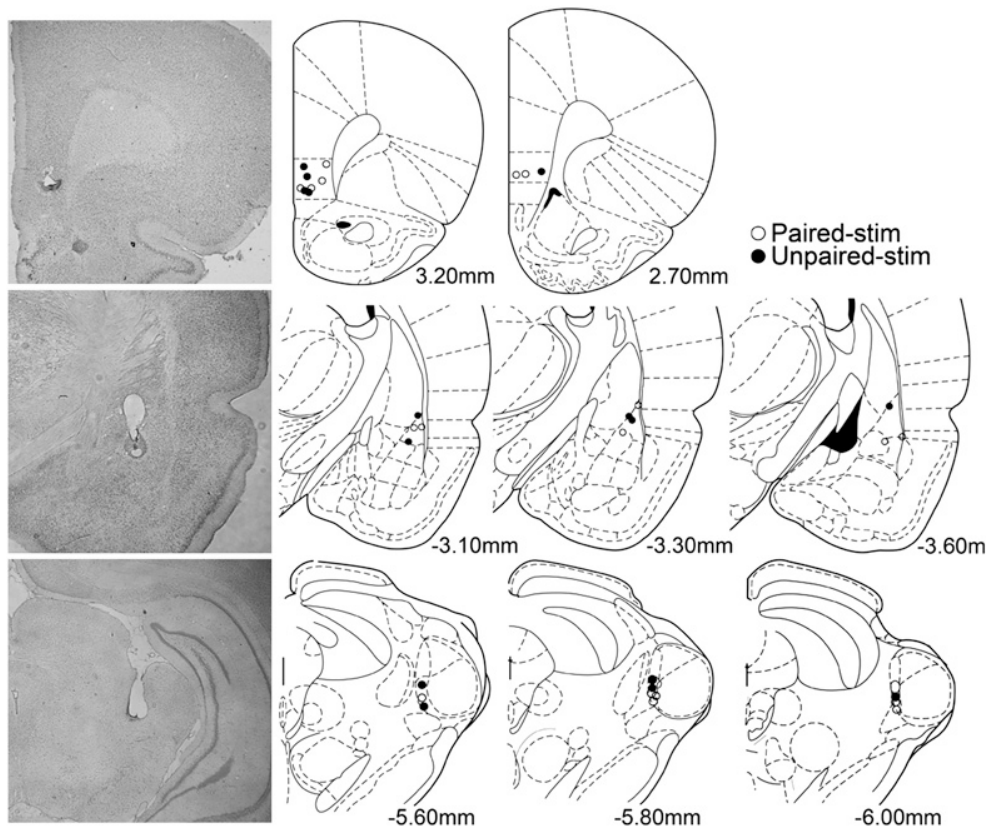

Figure 5. Histological verification of the stimulating and recording sites for experiment 5 . Each animal was implanted with two stimulating electrodes in the IL and MGm and a recording electrode in the LA for EFP recording. The photomicrographs in the left column show representative coronal sections of the IL (top), LA (middle), and MGm (bottom). The diagram in the right column displays the location of all stimulating and recording electrodes mapped onto the matching sections in the atlas (modified from Paxinos and Watson 1998 and reprinted with permission from Elsevier (1)1998).

condition for inducing such synaptic and behavioral changes. In addition, the current study provides an intriguing possibility that cellular changes induced during anesthesia can be transferred to behavioral modification of a previously learned fear response.

\section{Timed activation of the mPFC is necessary for extinction}

The mPFC, especially the IL, plays an essential role in fear extinction. Previous studies have shown that extinction training enhances the intrinsic neuronal excitability of the IL (Santini et al. 2008). Our data also showed that Fos expression was significantly increased in the IL following extinction training. In addition, an equivalent level of Fos expression was found in the PL. Although these data suggest that both the PL and IL were active during the extinction, they are somewhat inconsistent with a popular model of $\mathrm{mPFC}$ function in which opposite roles were assigned for the PL and IL: the former in expression and the latter in suppression of conditioned fear memory. This "PL-IL model" is supported by multiple studies showing antagonistic interactions between the two mPFC subregions. PL stimulation given paired with the CS during extinction training significantly delayed extinction learning, while IL simulation facilitated extinction (Vidal-Gonzalez et al. 2006). A unit-recording study showed neuronal activities related to extinction memory and its recall in the IL, but not in the PL (Milad and Quirk 2002). IL, but not PL, neurons displayed enhanced intrinsic excitability after extinction training (Santini et al. 2008). In addition, PL neurons showed conditioned responses to the tone CS that diminished with extinction (BurgosRobles et al. 2009).

However, contradictory evidence also exists. Most of all and most relevant to our result, a set of immunohistochemistry data with an almost identical method as ours has shown indistinguishable Fos expressions in both PL and IL after extinction training (Santini et al. 2004). Such activation patterns may come from two different events: fear expression and extinction. A typical extinction session unavoidably involves retrieval of fear memory, at least in the beginning of the session. The PL might be activated at the retrieval phase. It is universally accepted that fear-conditioned animals show robust freezing during the early phase of an extinction session. Only the trials in the later phase of the extinction training are directly involved in extinction and may activate IL. Considering the limited time resolution of Fos immunochemistry, which was applied almost immediately after extinction training, the common pattern of activation in PL and IL may actually represent two temporally separate events.

Another interpretation of the Fos expression in the PL could be found in the literature outside fear conditioning. The PL and IL are synergistically engaged in a situation where a discontinuity of the context must be detected to generate an appropriate response. For example, previous studies using spatial memory tasks found that mPFC is engaged when shifting behavioral strategies becomes necessary to cope with changes in the experimental procedure (Gisquet-Verrier and Delatour 2006) or when memory retrieval is requested in an altered contextual condition (Jo et al. 2007). The tone-CS in extinction represents a similar challenge in which its informational significance is reversed from acquisition (i.e., from danger to safety signal). These models suggest that the role of the PL might be "interchangeable with" rather than "contradictory to" that of the IL in some situations.

Fos expression in the mPFC was blocked in anesthetized rats even after the brain-stimulation CS had been delivered repeatedly, a procedural manipulation equivalent to extinction training in conscious animals. It is perhaps no coincidence that the repeated presentation of the CS also failed to reduce the fear CR measured in the awake state. One possibility is that the massive inhibition due to anesthesia diminished the reactivity of the sensory network and consequently blocked the auditory CS transmission from reaching the amygdala. In fact, general anesthetics, including sodium pentobarbital, are known to inhibit auditory transmission by altering neuronal activities in the inferior colliculus (Kuwada et al. 1989), the auditory thalamus (Wan and Puil 2002), and the auditory cortex (Gaese and Ostwald 2001). To circumvent this problem, we employed the brain-stimulation CS, utilizing the known direct connection between the MGm and the LA. Robust EFPs to the MGm stimulation were recorded in LA neurons, indicating that the CS reached the amygdala. The EFPs also displayed constant latency (Table 1). Yet, repeated delivery of the brain-stimulation CS to the LA failed to induce behavioral extinction. These results, together with the data showing sparse Fos expression in the anesthetized animals, suggest that the lack of behavioral extinction might be attributable to the suppressed function of the mPFC.

One might argue that the failure to show an extinction effect in anesthetized rats was due to state dependency. Retrieval of extinction memory is sensitive to the contextual change (Bouton 2004), and extinction training under a drugged state was not effective when tested under drug-free conditions (Bouton et al. 1990). Anesthesia provides a salient internal state that could constitute a distinct context. It is possible that extinction memory 
A

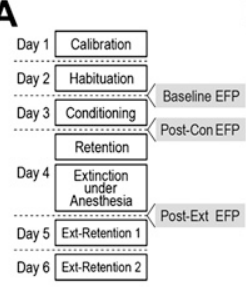

B

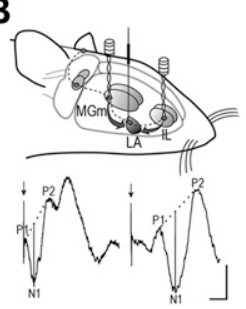

D

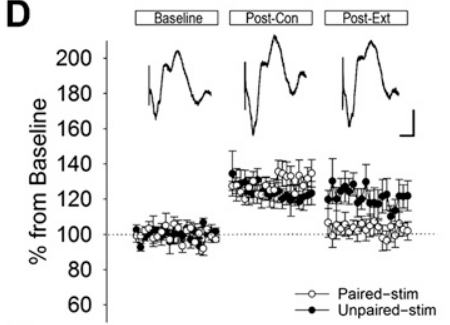

$\mathbf{F}$

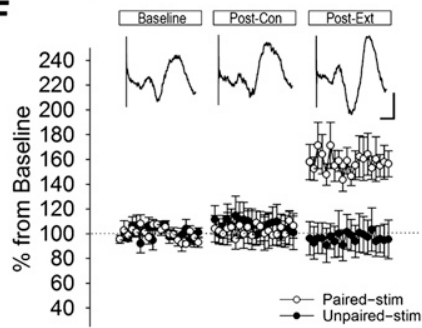

C

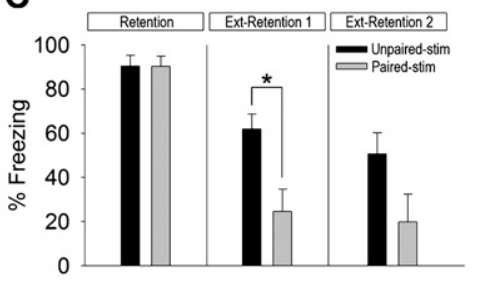

E
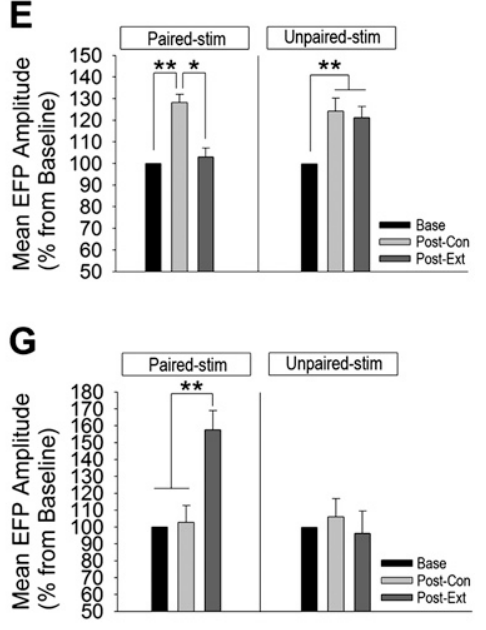

Figure 6. Alterations of MGm-LA and IL-LA synapses following extinction training under anesthesia. $(A)$ Experimental procedure. The training and testing procedures were identical to those used in experiment 4 (Fig. 1D) except that three EFP recording sessions were inserted after habituation, fear conditioning, and extinction training under anesthesia. (B) Schematic drawing of the rat brain showing the arrangement of stimulating and recording electrodes (top) and representative EFP waveforms recorded in the LA evoked by the MGm (bottom left) or IL (bottom right) stimulation. The amplitude of EFP waveform was defined as the distance from the first negative peak (N1) to the line that connects the first (P1) and the second (P2) positive peak (solid line). Arrows indicate stimulation onset. (C) The rate of CR measured across different sessions. Although the two groups acquired equivalent levels of CRs after conditioning, as evidenced by the equally high level of freezing during the retention session, rats in the Paired-stim group showed significantly lower levels of freezing than the Unpaired-stim group following the extinction training under anesthesia. (D) Recorded EFPs in the LA to the MGm stimulation. Data points are across two-trial blocks, represented as a Mean \pm SEM percentage relative to the baseline. Superimposed are representative waveforms of the Paired-stim group at each recording session. (E) Mean EFP amplitudes in the MGm-LA pathway. EFPs were significantly increased after conditioning in both groups as evidenced by marked differences between Base and Post-Con. The two groups differ, however, in that only the Paired group showed significant reduction in EFP amplitude in the Post-Ext test. $(F)$ Recorded EFPs in the LA to the IL stimulation. $(G)$ Mean EFP amplitudes in the IL-LA pathway. Fear conditioning did not alter the mean EFP amplitude of the IL-LA response from the baseline (no difference between Base and Post-Con for both groups). However, the Paired-stim group showed significantly enhanced EFPs in the Post-Ext session, whereas the EFPs of Unpaired-stim group remained unchanged. Scale bars $=10 \mathrm{msec}, 200 \mu \mathrm{V} .{ }^{\star} P<0.05,{ }^{* \star} P<0.01$.

was not retrieved because the training and testing conditions were different. However, the state-dependency account can be disputed by our data: IL stimulation, a local brain activation, which is certainly not sufficient to reverse the anesthetized state; and rescued behavioral extinction, implying that the different level of consciousness does not fully explain the lack of extinction effect. Therefore, the lack of extinction effect cannot be attributable to the contextual change between extinction training and retention testing.

Consistent with converging lines of evidence, our data provide unique evidence supporting the necessity of mPFC activation in extinction. In the current study, a "surrogate" activity, the electrical stimulation of the $\mathrm{mPFC}$, was able to rescue the extinction deficit caused by anesthesia. The surrogate activity must be appropriately timed in relation to the CS, because only the Pairedstim group showed reduction of the fear CR (Fig. 6C), demon- strating a striking similarity to the facilitatory effect of mPFC stimulation on normal extinction (Milad and Quirk 2002). Therefore, it can be argued that mPFC activity during extinction training is essential for the development of synaptic changes needed to suppress the CR.

\section{Reversal of conditioning-induced synaptic enhancement in the MGm-LA pathway underlies extinction}

The conditioning-induced enhancement of synaptic efficacy in the MGm-LA pathway returned to the baseline level after extinction training under anesthesia. The reversal of neural plasticity following extinction training has been reported in previous studies. Firing rates of LA neurons increased after conditioning and then decreased after extinction (Quirk et al. 1995). A study using fMRI reported a similar temporal pattern of activation in the amygdala (LaBar et al. 1998). In addition, neuronal activity in the LA was decreased in response to the extinguished CS in a context-specific manner (Hobin et al. 2003). Our data also showed that conditioning-induced synaptic potentiation was reversed following extinction training, accompanied by behavioral suppression of the fear CR. Therefore, excitability of LA neurons seems to be closely linked to the acquisition and extinction of the fear response.

A possible mechanism for the reversal in the MGm-LA synaptic plasticity is depotentiation. The weight of the evidence supports this hypothesis. EPSCs to thalamic stimulation was depressed after extinction training when measured ex vivo, and the extinction training also reversed the surface expression level of AMPA-receptor subunits, providing a cellular basis for synaptic depotentiation (Kim et al. 2007). Similarly, low frequency stimulation in the external capsule induced depotentiation of an LA neuronal response (Lin et al. 2003). Recent experimental evidence suggests that enhanced activity of local interneurons contributes to the extinction-induced synaptic depotentiation. For example, Chhatwal et al. (2005) showed that gephyrin, a $\mathrm{GABA}_{\mathrm{A}}$ receptor clustering protein, was significantly increased after extinction, together with the increased number of available $\mathrm{GABA}_{\mathrm{A}}$ receptors at the cell surface. Likewise, Lin et al. (2009) reported that $\mathrm{GABA}_{\mathrm{A}}$ receptor-mediated miniature IPSCs, as well as $\mathrm{GABA}_{\mathrm{A}}$ receptor insertion were greatly increased after extinction. Interestingly, inspired by powerful inhibitory control of the local interneurons over LA projection neurons (Rosenkranz and Grace 2002a), Li et al. (2008) have tested a computational model in which enhanced activity of the LA interneurons and strengthening of their connections to the neighboring pyramidal neurons suppressed the activity of the projection neurons. In parallel with the changes in the interneurons, the model also proposed synaptic depression of the excitatory projection neurons. 
Based on these studies and our own data, we suggest that the longterm extinction memory is dependent on the modification of the MGm-LA synapses, especially those encoding the initial fear memory, and that the local inhibitory circuit might mediate the conversion of the potentiated synapses.

Depotentiation of the thalamic input is at odds with some of the behavioral data. Well-established results from spontaneous recovery, context-dependent renewal, and reinstatement support the fragile nature of extinction memory: Extinguished CRs often return almost instantly in those behavioral paradigms (Myers and Davis 2007). It is difficult to conceive a molecular mechanism that would revive the synaptic strength that has been once depotentiated within such a short time. This suggests that there must be other circuits that store permanent fear memory (including a different population of LA neurons), which can be switched on and off relatively quickly. In fact, some LA neurons have been found to be activated persistently to the CS even after extinction training (Repa et al. 2001). The mPFC might regulate the activation of those neurons after extinction (Herry et al. 2008). In addition, synaptic potentiation of the IL-LA pathway observed in the current result suggests that the functional connectivity between IL and LA increases after extinction training, thereby allowing the $\mathrm{mPFC}$ to exert a regulatory control over the activities of LA neurons with higher fidelity.

\section{LTP-like changes in the IL-LA pathway accompany extinction}

One of the most significant findings of our study is the enhanced synaptic efficacy in the IL-LA pathway after extinction training. If extinction requires synaptic enhancement of IL-LA pathway, a crucial question arises: How is it translated into inhibitory actions within the amygdala?

One possibility is that synaptic potentiation is induced within a subset of LA neurons. The LA has a heterogeneous neuronal composition, and different subnuclei play distinct roles in the acquisition and extinction. For instance, Repa et al. (2001) have found heterogeneous neuronal populations that differ in their firing patterns during the acquisition and extinction phases. Furthermore, Herry et al. (2008) reported a subset of neurons in the basolateral nucleus of the amygdala. These "extinction neurons" displayed an increased firing rate in response to the CS during the extinction training as opposed to "fear neurons," which fired increasingly during and continuously after fear conditioning. Interestingly, the extinction neurons have dense reciprocal connections with the $\mathrm{MPFC}$, whereas the fear neurons lack such connections. Therefore, IL stimulation is likely to recruit extinction, but not fear neurons. It is possible that the EFPs to the IL stimulation reflect the activation of the extinction neurons, and synaptic potentiation might be induced in the selective subclass of LA neurons by the extinction training. This subset of LA neurons might regulate fear response by changing the activities of other neurons in the LA or those in other amygdala subregions that project more directly to the output areas outside the amygdala.

Other hypotheses propose that synaptic potentiation of the IL-LA pathway involves inhibitory interneurons. In one, Rosenkranz and Grace (2002a) have argued that prefrontal regulation of the BLA is mediated by the interneurons, demonstrating that hyperpolarization of the BLA projection neurons was caused by the intrinsic GABAergic current. Activation of the inhibitory interneurons by prefrontal stimulation is in stark contrast with sensory cortical inputs, which drive the projection neurons. Following this scenario, it is plausible to assume that LTP-like synaptic changes in the LA lead to excitability increase in the local inhibitory circuit and underlie the suppression of the fear CR, reflecting unique functional organization of the IL-LA pathway. Caution must be taken in interpreting the stimulation-induced neural response in LA as the data may reflect antidromic activation of some LA neurons. Reciprocal connections between the mPFC and the LA have been firmly established (McDonald 1998). Recording from BLA neurons, Likhtik et al. (2005) have suggested that mPFC stimulation might activate BLA projection neurons antidromically and, in turn, recruit feedback interneurons via the local axon collaterals. The net result is the suppression of BLA projection neurons through feedback inhibition evoked by backfiring. If that is the case, the potentiated EFP to IL stimulation is more likely to involve enhanced excitability of the projection neurons rather than that of the interneurons. It is difficult to determine whether the enhanced IL-LA response reflects orthodromic and/or antidromic propagation of the stimulation without further experiments tailored to address such a question. A limited conclusion can still be drawn, however, based on the current data: Repeated coactivation of MGm and IL strengthens, rather than weakens, LA neuronal excitability to the IL stimulation. How such change is induced and translated into fear suppression would require further knowledge on the functional dynamics of the fronto-amygdalar circuit in relation to emotional regulation.

In sum, our data demonstrate that CS-specific fear expression is significantly reduced and preceded by long-term synaptic changes in the two input pathways into the LA: the reversal of conditioning-induced synaptic potentiation in the MGm-LA pathway and the enhanced synaptic strength of the IL-LA pathway. Our data also confirmed that the mPFC regulates the extinction process and possibly initiates the critical synaptic changes within the LA. The synaptic changes did not necessarily require conscious processing of the CS as all the extinction procedures were administered to anesthetized rats. Due to the mechanical nature of the current stimulation protocol, our data bridge the gap between synaptic plasticity, which has been typically studied in slice preparation; and behavioral learning, which can be assessed only in fully conscious animals. Although our data strongly suggest that synaptic changes in the critical brain circuit are sufficient to form a new or modify the existing memory regardless of how they were induced, further studies are required to elucidate the cellular and molecular mechanisms and their direct mapping to fear memory. The reduced freezing observed in the current study may not have resulted from the same network dynamics as those that might be activated in awake animals.

\section{Materials and Methods}

\section{Experiment 1}

\section{Subjects}

Male Sprague-Dawley rats (260-320 g; Orient Bio) were housed individually in a temperature- and humidity-controlled environment $\left(22^{\circ} \mathrm{C} \pm 2{ }^{\circ} \mathrm{C}\right.$ and $50 \% \pm 10 \%$, respectively) with ad libitum access to food and water. They were maintained on a 12-h light/ dark cycle. All behavioral procedures were conducted during the dark phase of the cycle.

\section{Experimental procedure}

Rats were assigned to either the anesthetized-extinction (Anesthetized-Ext, $n=6$ ) or anesthetized-control (Anesthetized, $n=6$ ) groups. On the day of conditioning, they were placed in a distinct context (Context A) and allowed a 3-min acclimation period. Rats then received Pavlovian fear conditioning consisting of five pairings of a tone-CS $(4 \mathrm{kHz}, 80 \mathrm{~dB})$ and a footshock-US $(0.5 \mathrm{~mA}, 0.5 \mathrm{msec})$ with a randomized intertrial interval averaged at $180 \mathrm{sec}$, ranging from 150 to $210 \mathrm{sec}$. Two minutes after the termination of the fifth US, rats were returned to their home cages. On the next day, the rats were tested for retention of fear memory in a novel context (Context B) and then immediately anesthetized 
with sodium pentobarbital injection ( $42 \mathrm{mg} / \mathrm{kg}$, i.p.). Under the anesthetized state, the Anesthetized-Ext group received $15 \mathrm{CS}$ presentations, whereas the Anesthetized group stayed in the same context for the same duration without any CS presentation. After $24 \mathrm{~h}$, all rats were put into Context B and exposed to $10 \mathrm{CS}$-alone trials to measure freezing as an index of the fear CR. Freezing was defined as total absence of any movement, except that required for respiration. Freezing was measured by an experienced observer on recorded video using a stopwatch.

\section{Behavioral apparatus}

All behavioral procedures were undertaken in a black Plexiglas chamber $(30 \mathrm{~cm} \times 25 \mathrm{~cm} \times 25 \mathrm{~cm})$ placed in a sound-attenuating cubicle $(48 \mathrm{~cm} \times 55 \mathrm{~cm} \times 45 \mathrm{~cm})$, and the chamber was equipped with a video camera and a speaker $(8 \mathrm{~cm} \times 4 \mathrm{~cm}, 8 \Omega)$ mounted overhead. The CS and US were delivered automatically using a customized program built on LabVIEW (National Instruments), and the animals' behaviors were videotaped for off-line analysis. Throughout the entire procedure, rats were conditioned in Context $\mathrm{A}$ and extinction-trained and tested in Context B. Context A was illuminated by orange bulbs and floored with a grid composed of 16 stainless steel rods $(0.5 \mathrm{~cm}$ in diameter, $1.5 \mathrm{~cm}$ apart) connected to a shock scrambler and generator (Coulbourn Instruments) for electrical footshock deliverance. Context B was lit by blue bulbs, with a flat floor covered with wood shavings. At the termination of each behavioral session, the chamber and the grid were cleaned with $70 \%$ ethanol solution.

\section{Experiment 2}

\section{Surgery}

Rats were anesthetized with sodium pentobarbital (60 mg/kg, i.p.) with supplemental doses injected as needed and mounted on a stereotaxic apparatus (David Kopf Instruments). The scalp was incised and retracted to expose the skull, and the head position was adjusted to place bregma and lambda on the same horizontal plane. Small burr holes were drilled, and the dura overlying the targeted brain region was removed for the chronic implantation of a stimulating electrode. For electrical brain stimulation, a bipolar stainless steel electrode (Plastics One) insulated with polyimide (tip diameter, $0.125 \mathrm{~mm}$; impedance, $15-30 \mathrm{k} \Omega$ ) except for the tip, was targeted $5.6-\mathrm{mm}$ posterior, $2.9-\mathrm{mm}$ lateral, and $5.5-\mathrm{mm}$ ventral to bregma to place the electrode in the MGm of the left hemisphere of the brain (Paxinos and Watson 1998). The electrode was secured with anchoring screws and dental cement after being placed onto the target region. Rats were allowed at least $7 \mathrm{~d}$ to recover, during which they were handled daily.

\section{Experimental procedure}

Rats were divided into the MGm-stim $(n=8)$ or Anesthetized groups $(n=8)$ and underwent the same procedure except that the MGm stimulation was used as the CS instead of an auditory tone (see Fig. 1D). Rats in the MGm-stim group were exposed to 15 presentations of the CS, MGm stimulation, during the extinction training under anesthesia. To use the MGm stimulation as the CS, two preliminary processes were needed to individually adjust the current intensity level. On the first day, test stimuli were given gradually increasing the current intensity from $50 \mu \mathrm{A}$ up to $150 \mu \mathrm{A}$ in steps of $20 \mu \mathrm{A}$ to meet the optimal intensity level where stimulus-specific orienting behaviors were observed without any fear responses, a procedure termed as "Calibration." On the following day, rats were exposed to three MGm-stimulation CSs with an adjusted current intensity level to assure that the MGm stimuli per se elicited no fear responses, a procedure termed as "Habituation."

\section{Electrical stimulation}

A bipolar stimulating electrode was used to deliver the brainstimulation CS (MGm electrode). The stimulation parameters were as shown in Figure 1B. Thirty intermittent trains (100-msec duration, 1-sec intertrain interval) of monophasic square pulses
(100- $\mu$ sec duration, $100 \mathrm{~Hz}$ ) were generated by a stimulator (SYSA300; World Precision Instruments). The output of the stimulator was connected to a stimulus isolator (A360, WPI), which maintained the current intensity of MGm stimulation at an individually adjusted level $(50-150 \mu \mathrm{A})$.

\section{Experiment 3}

\section{Surgery}

Using the surgical procedure described in experiment 2, all rats were implanted with a bipolar stainless steel electrode in the MGm in order to convey electrical stimulation as the CS.

\section{Experimental procedure}

We compared the Fos expression level in the mPFC of rats that received extinction training in either an awake or an anesthetized state. Three groups of rats were fear conditioned using MGm stimulation as the CS, and two out of the three conditioned groups received extinction training on the following day in either an awake or anesthetized state (Ext, $n=5$ and Anesthetized-Ext, $n=$ $5)$; the remaining group did not receive extinction training (NoExt, $n=4$ ). To compare the number of Fos-immunoreactive (IR) neurons affected by nonmnemonic factors, two control groups (Naïve, $n=4$ and Stimulation-Only, $n=5$ ) were added. The Naïve group received the same duration of exposure to the context as the Ext group, receiving neither CS nor US. The Stimulation-Only group received the same number of MGm stimulations but never received the US. Ten minutes after the completion of the whole procedure, a final test CS was delivered, and $70 \mathrm{~min}$ later, rats were sacrificed to evaluate Fos expression.

\section{Fos immunohistochemistry and cell counting}

Seventy minutes after being presented with the final test CS, rats were deeply anesthetized with sodium pentobarbital $(80 \mathrm{mg} / \mathrm{kg}$, i.p.) and perfused transcardially with PBS followed by $4 \%$ paraformaldehyde in PBS, $\mathrm{pH}$ 7.4. Brains were then extracted, postfixed overnight at $4{ }^{\circ} \mathrm{C}$ in the same fixative, and transferred to $30 \%$ sucrose in PBS for cryoprotection. Brains were cut in coronal sections $(16-\mu \mathrm{m}$ thick) with a freezing microtome (SM $2000 \mathrm{R}$; Leica). After washing free-floating sections in Tris buffered saline (TBS), they were pretreated with a solution containing $3 \%$ bovine serum albumin and $0.2 \%$ Triton X-100 in TBS (TBST) for $60 \mathrm{~min}$ to block unspecific antibody binding. The sections were then incubated with primary rabbit anti-c-fos antibody (1:1000; Calbiochem) for $60 \mathrm{~min}$ at room temperature. After washing in TBST three times, the sections were placed in cyanine 3 (Cy3) goat-antirabbit IgG (1:1000; Invitrogen) containing blocking solution for $60 \mathrm{~min}$. The sections were rinsed in TBST three times and stained with 4',6'-diamidino-2-phenylindole (DAPI) $(10 \mu \mathrm{g} / \mathrm{mL}$ in TBS) for $10 \mathrm{~min}$ to counterstain nuclei. After washing, the sections were coverslipped with aqueous mounting medium (Biomeda).

For quantitative analysis, the regions of interest, infralimic (IL) and prelimbic (PL) regions of the mPFC, were chosen with regard to abundant data suggesting that they were essential for consolidation of fear extinction memory and its retrieval, and the M1 region of motor cortex was also analyzed for the control of unspecific Fos expression in irrelevant brain regions. Images of each region were obtained with an Axioskop 2 epifluorescent microscope (Zeiss) equipped with a CoolSNAP HQ digital camera (Roper Scientific) and MetaView software (Universal Imaging). The light level and camera exposure times for each sample were constantly maintained. Fluorescence was visualized with a UV filter (365-nm excitation) for DAPI and a green filter (546-nm excitation) for $\mathrm{Cy} 3$, and then two subsequent pictures were imported separately to Adobe Photoshop 7.0 (Adobe Systems) and superimposed. Two images $(200 \times 200 \mu \mathrm{m})$ were obtained from each animal for cell counting in the PL, IL, and M1. Fos-IR cells were counted by an experimenter blind to the behavioral conditions. All neurons revealed by DAPI staining (blue) were counted and those with Fos-IR labeling revealed by Cy3 (red) were detected. A neuron was considered as a Fos-IR neuron if $\mathrm{Cy} 3$ labeling is found within the nucleus because the immediate early 
genes function as transcription factors in the nucleus. The ratio of Fos-IR neurons to DAPI-labeled neurons was then calculated per image. Two ratios were calculated and the mean was obtained per each animal.

\section{Experiment 4}

\section{Surgery}

Rats were implanted with two bipolar stainless steel electrodes in the MGm and the IL via the surgical procedure described above. Both the MGm and IL electrodes were located in the left hemisphere of the brain. The IL electrode was placed $2.9-\mathrm{mm}$ anterior, $0.5-\mathrm{mm}$ lateral, and $5.0-\mathrm{mm}$ ventral to bregma (Paxinos and Watson 1998).

\section{Experimental procedure}

Rats were divided into Paired-stim $(n=10)$ and Unpaired-stim groups $(n=9)$. Rats in both groups underwent the same experimental procedure as in the second experiment except that IL stimulation was applied during the extinction training under anesthesia. Rats were fear conditioned with MGm stimulation as the CS. On the following day, they were presented with three CSonly trials to test the retention of the conditioned fear memory. Immediately after the retention test, rats were anesthetized and underwent extinction training. The Paired-stim group received 15 trials of MGm and IL stimulation in a paired manner, and IL stimulation was initiated $50 \mathrm{msec}$ after the onset of $\mathrm{MGm}$ stimulation. The Unpaired-stim group received the same number of MGm and IL stimulation, but they were arranged apart from each other with randomized intervals longer than 60 sec.

\section{Electrical microstimulation}

The two stimulating electrodes were used to deliver brain-stimulation CS (MGm electrode) and to facilitate neuronal activity in the IL region (IL electrode). Stimulation parameters were identical for both electrodes (see Fig. 1B,C). Thirty intermittent trains (100msec duration, 1-sec intertrain interval) of monophasic square pulses $(100-\mu$ sec duration, $100 \mathrm{~Hz})$ were generated by a stimulator (SYS-A300; World Precision Instruments). The output of the stimulator was connected to two stimulus isolators (A360, WPI) which maintained the current intensity of MGm stimulation at individually adjusted levels $(50-150 \mu \mathrm{A})$ and IL stimulation constantly at $100 \mu \mathrm{A}$.

\section{Experiment 5}

\section{Surgery}

For the EFP recording, a sharp tungsten electrode $(125 \mu \mathrm{m}$ in diameter, impedance, $4-5 \mathrm{M} \Omega$, A-M systems) was stereotaxically positioned in the LA (3.2-mm anterior, $5.3-\mathrm{mm}$ lateral to bregma, and $\sim 6.0-\mathrm{mm}$ ventral from dura) with two stimulating electrodes placed in the MGm and IL according to the coordinates described above (Paxinos and Watson 1998). All the electrodes were located ipsilaterally in the left hemisphere of the brain. The dorsoventral placement of the stimulating and recording electrodes was adjusted to maximize the MGm/IL-evoked field potentials in the LA. After being placed in the target regions, they were secured with anchoring screws and dental cement, and a reference electrode consisting of a bundle of stainless steel wires was affixed to the skull through the anchoring screws.

\section{Experimental procedure}

Rats were assigned to either the Paired-stim $(n=6)$ or Unpairedstim $(n=5)$ groups and went through the identical experimental schedule as their counterparts in the fourth experiment except that EFP recordings were performed $14 \mathrm{~h}$ after each behavioral session in both the MGm-LA and IL-LA pathways.

\section{Electrophysiology}

All electrophysiological recordings were performed under anesthesia induced by sodium pentobarbital. Low-frequency mono- phasic test pulses (100- $\mu$ sec duration, $0.4-0.9 \mathrm{~mA}, 0.1 \mathrm{~Hz})$ were applied either to the MGm or IL to evoke extracellular field potentials in the LA. Stimulation applied to these two sites generated distinct responses in the LA (see Fig. 6B). The EFPs were amplified $(\times 1000)$, filtered $(1 \mathrm{~Hz}-1 \mathrm{kHz})$, digitized $(10 \mathrm{kHz})$, and displayed on an oscilloscope for real-time monitoring and stored in a personal computer for off-line analysis via a customized program built on LabVIEW. The dorsoventral location of the recording electrode was adjusted so that the waveforms generated by MGm stimulation met the criterion previously suggested for the MGm-LA circuit (Clugnet and LeDoux 1990; Rogan and LeDoux 1995; Yaniv et al. 2001). Once the electrode positions were determined and electrodes secured in the MGm and the LA, the IL electrode was positioned in a similar manner. The waveform recorded in the IL-LA circuit in previous studies was used as a criterion (Rosenkranz and Grace 1999; Maroun 2006). Thirty minutes after the final positioning of the stimulating electrode in the IL, baseline EFPs were recorded by applying 40 test pulses at every $30 \mathrm{sec}$ for $20 \mathrm{~min}$ with current intensity individually adjusted to generate approximately half-maximal amplitudes (0.2-1 $\mathrm{mA}$ for MGm and 0.5-1.5 mA for IL stimulation).

Following $7 \mathrm{~d}$ of recovery, animals were anesthetized with sodium pentobarbital, and an additional baseline recording for EFP was obtained in the same manner to test the maintenance of waveforms in each pathway. The stimulation intensity was adjusted to a level that produced the same waveform recorded on the day of surgery. More recordings were taken, one at $14 \mathrm{~h}$ after the conditioning session and another after the extinction session. For quantitative analysis, the amplitudes of the MGm-LA and IL-LA EFPs were defined as shown in Figure 6B. The ratio of each EFP amplitude value to the mean EFP amplitude of baseline was calculated, and two percentile values were integrated as a mean, yielding 20 data points per each pathway in a recording session. The mean latency of the first negative peak (N1) of EFP waveforms was measured in each pathway (Fig. 6B). Only the electrophysiological data from rats that showed (1) correct placements of both MGm/IL-stimulating electrodes and a recording electrode in the LA (Fig. 5) and (2) stable EFPs over all recording sessions were included for further analysis. Out of the initial 15 implanted rats, 11 fulfilled the criteria and were successfully recorded over $10 \mathrm{~d}$ including the recovery period.

\section{Histology}

After the completion of experiments, marking lesions were made by passing direct current $(0.1 \mathrm{~mA}, 10 \mathrm{sec})$ through the electrodes, and histological verification of the electrode placements was performed. All animals were deeply anesthetized with an overdose injection of sodium pentobarbital $(80 \mathrm{mg} / \mathrm{kg}$ i.p. $)$ and were transcardially perfused with $0.9 \%$ saline, followed by a $10 \%$ formaldehyde solution. After extraction, the brains were postfixed in the same fixative overnight, transferred to $30 \%$ sucrose in $10 \%$ formaldehyde solution, and were stored at $4^{\circ} \mathrm{C}$ for several days until they sank to the bottom. They were cut into $50 \mu \mathrm{m}$ coronal sections with a freezing microtome (SM 2000 R; Leica). The sections were mounted on gelatin-coated slides, stained with cresyl violet, and coverslipped with Permount, and examined under light microscopy. Only data from rats with precise placements of the electrodes as targeted were included in the analysis.

\section{Statistical analysis}

For behavioral experiments, group comparisons for freezing levels were analyzed with repeated-measures ANOVA, whereas group differences for mean freezing levels in the second extinctionretention session and mean Fos-IR counts were determined via Student's $t$-tests or one-way ANOVA. All tests were two-tailed. A Bonferroni's post-hoc test was followed for analysis in which more than three groups were taken for comparisons. The mean EFP amplitudes and peak latencies were compared using repeatedmeasures ANOVA. A Bonferroni's post-hoc test was followed to further analyze group effects within each session or session effects within each group. Only $P<0.05$ was considered to be statistically significant. All data were reported as mean and SEM. 


\section{Acknowledgments}

We thank Yong Sang Jo, Drs. Il Hwan Kim and Hyun Kim for c-Fos data acquisition, Drs. Mark E. Bouton, J. Amiel Rosenkranz, and Sukwoo Choi for helpful comments on this manuscript, and Jeong-Tae Kwon and Ingie Hong for critical discussions. This work was supported by a Korea Science and Engineering Foundation (KOSEF) grant (20090081145) funded by the Korea government (MEST) and Cognitive Neuroscience Program of the Korea Ministry of Science and Technology (M10644020003-06N4402-00310).

\section{References}

Bauer EP, LeDoux JE. 2004. Heterosynaptic long-term potentiation of inhibitory interneurons in the lateral amygdala. J Neurosci 24: 95079512.

Blair HT, Schafe GE, Bauer EP, Rodrigues SM, LeDoux JE. 2001. Synaptic plasticity in the lateral amygdala: A cellular hypothesis of fear conditioning. Learn Mem 8: 229-242.

Bouton ME. 2004. Context and behavioral processes in extinction. Learn Mem 11: 485-494.

Bouton ME, Kenney FA, Rosengard C. 1990. State-dependent fear extinction with two benzodiazepine tranquilizers. Behav Neurosci 104: 44-55.

Burgos-Robles A, Vidal-Gonzalez I, Quirk GJ. 2009. Sustained conditioned responses in prelimbic prefrontal neurons are correlated with fear expression and extinction failure. J Neurosci 29: 8474-8482.

Chhatwal JP, Myers KM, Ressler KJ, Davis M. 2005. Regulation of gephyrin and $\mathrm{GABA}_{\mathrm{A}}$ receptor binding within the amygdala after fear acquisition and extinction. J Neurosci 25: 502-506.

Clugnet MC, LeDoux JE. 1990. Synaptic plasticity in fear conditioning circuits: Induction of LTP in the lateral nucleus of the amygdala by stimulation of the medial geniculate body. J Neurosci 10: 2818-2824.

Davis M, Rainnie D, Cassell M. 1994. Neurotransmission in the rat amygdala related to fear and anxiety. Trends Neurosci 17: 208-214.

Falls WA, Miserendino MJ, Davis M. 1992. Extinction of fear-potentiated startle: Blockade by infusion of an NMDA antagonist into the amygdala. J Neurosci 12: 854-863.

Fanselow MS, LeDoux JE. 1999. Why we think plasticity underlying Pavlovian fear conditioning occurs in the basolateral amygdala. Neuron 23: $229-232$

Gaese BH, Ostwald J. 2001. Anesthesia changes frequency tuning of neurons in the rat primary auditory cortex. J Neurophysiol 86: 10621066.

Gisquet-Verrier P, Delatour B. 2006. The role of the rat prelimbic/infralimbic cortex in working memory: Not involved in the short-term maintenance but in monitoring and processing functions. Neuroscience 141: 585-596.

Gold PE, Weinberger NM, Sternberg DB. 1985. Epinephrine-induced learning under anesthesia: Retention performance at several trainingtesting intervals. Behav Neurosci 99: 1019-1022.

Herry C, Garcia R. 2002. Prefrontal cortex long-term potentiation, but not long-term depression, is associated with the maintenance of extinction of learned fear in mice. J Neurosci 22: 577-583.

Herry C, Mons N. 2004. Resistance to extinction is associated with impaired immediate early gene induction in medial prefrontal cortex and amygdala. Eur J Neurosci 20: 781-790.

Herry C, Trifilieff P, Micheau J, Luthi A, Mons N. 2006. Extinction of auditory fear conditioning requires MAPK/ERK activation in the basolateral amygdala. Eur J Neurosci 24: 261-269.

Herry C, Ciocchi S, Senn V, Demmou L, Muller C, Luthi A. 2008. Switching on and off fear by distinct neuronal circuits. Nature 454: 600-606.

Hobin JA, Goosens KA, Maren S. 2003. Context-dependent neuronal activity in the lateral amygdala represents fear memories after extinction. J Neurosci 23: 8410-8416.

Jo YS, Park EH, Kim IH, Park SK, Kim H, Kim HT, Choi JS. 2007. The medial prefrontal cortex is involved in spatial memory retrieval under partialcue conditions. J Neurosci 27: 13567-13578.

Kaczmarek L, Robertson HA. 2002. Immediate early genes and inducible transcription factors in mapping of the central nervous system function and dysfunction. Elsevier, Amsterdam, The Netherlands.

Kim J, Lee S, Park K, Hong I, Song B, Son G, Park H, Kim WR, Park E, Choe $\mathrm{HK}$, et al. 2007. Amygdala depotentiation and fear extinction. Proc Natl Acad Sci 104: 20955-20960.

Kuwada S, Batra R, Stanford TR. 1989. Monaural and binaural response properties of neurons in the inferior colliculus of the rabbit: Effects of sodium pentobarbital. J Neurophysiol 61: 269-282.

Kwon JT, Choi JS. 2009. Cornering the fear engram: Long-term synaptic changes in the lateral nucleus of the amygdala after fear conditioning. J Neurosci 29: 9700-9703.
LaBar KS, Gatenby JC, Gore JC, LeDoux JE, Phelps EA. 1998. Human amygdala activation during conditioned fear acquisition and extinction: A mixed-trial fMRI study. Neuron 20: 937-945.

LeDoux JE, Cicchetti P, Xagoraris A, Romanski LM. 1990a. The lateral amygdaloid nucleus: Sensory interface of the amygdala in fear conditioning. J Neurosci 10: 1062-1069.

LeDoux JE, Farb C, Ruggiero DA. 1990b. Topographic organization of neurons in the acoustic thalamus that project to the amygdala. J Neurosci 10: 1043-1054.

LeDoux JE, Farb CR, Milner TA. 1991. Ultrastructure and synaptic associations of auditory thalamo-amygdala projections in the rat. Exp Brain Res 85: 577-586.

Lee HJ, Choi JS, Brown TH, Kim JJ. 2001. Amygdalar NMDA receptors are critical for the expression of multiple conditioned fear responses. J Neurosci 21: 4116-4124.

Li G, Nair SS, Quirk GJ. 2008. A biologically realistic network model of acquisition and extinction of conditioned fear associations in lateral amygdala neurons. J Neurophysiol 101: 1629-1646.

Likhtik E, Pelletier JG, Paz R, Pare D. 2005. Prefrontal control of the amygdala. J Neurosci 25: 7429-7437.

Likhtik E, Popa D, Apergis-Schoute J, Fidacaro GA, Pare D. 2008. Amygdala intercalated neurons are required for expression of fear extinction. Nature 454: 642-645.

Lin CH, Lee CC, Gean PW. 2003. Involvement of a calcineurin cascade in amygdala depotentiation and quenching of fear memory. Mol Pharmacol 63: 44-52.

Lin HC, Mao SC, Gean PW. 2009. Block of $\gamma$-aminobutyric acid-A receptor insertion in the amygdala impairs extinction of conditioned fear. Biol Psychiatry 66: 665-673.

Mao SC, Hsiao YH, Gean PW. 2006. Extinction training in conjunction with a partial agonist of the glycine site on the NMDA receptor erases memory trace. J Neurosci 26: 8892-8899.

Maren S, Quirk GJ. 2004. Neuronal signalling of fear memory. Nat Rev Neurosci 5: 844-852.

Maroun M. 2006. Stress reverses plasticity in the pathway projecting from the ventromedial prefrontal cortex to the basolateral amygdala. Eur J Neurosci 24: 2917-2922.

McDonald AJ. 1998. Cortical pathways to the mammalian amygdala. Prog Neurobiol 55: 257-332.

McKernan MG, Shinnick-Gallagher P. 1997. Fear conditioning induces a lasting potentiation of synaptic currents in vitro. Nature 390: 607611.

Milad MR, Quirk GJ. 2002. Neurons in medial prefrontal cortex signal memory for fear extinction. Nature 420: 70-74.

Milad MR, Vidal-Gonzalez I, Quirk GJ. 2004. Electrical stimulation of medial prefrontal cortex reduces conditioned fear in a temporally specific manner. Behav Neurosci 118: 389-394.

Myers KM, Davis M. 2007. Mechanisms of fear extinction. Mol Psychiatry 12: $120-150$.

Paxinos G, Watson C. 1998. The rat brain in stereotaxic coordinates. Academic, San Diego, CA.

Quirk GJ, Mueller D. 2008. Neural mechanisms of extinction learning and retrieval. Neuropsychopharmacology 33: 56-72.

Quirk GJ, Repa C, LeDoux JE. 1995. Fear conditioning enhances shortlatency auditory responses of lateral amygdala neurons: Parallel recordings in the freely behaving rat. Neuron 15: 1029-1039.

Quirk GJ, Russo GK, Barron JL, Lebron K. 2000. The role of ventromedial prefrontal cortex in the recovery of extinguished fear. J Neurosci 20: 6225-6231.

Repa JC, Muller J, Apergis J, Desrochers TM, Zhou Y, LeDoux JE. 2001. Two different lateral amygdala cell populations contribute to the initiation and storage of memory. Nat Neurosci 4: 724-731.

Rodrigues SM, Farb CR, Bauer EP, LeDoux JE, Schafe GE. 2004. Pavlovian fear conditioning regulates $\mathrm{Thr}^{286}$ autophosphorylation of $\mathrm{Ca}^{2}+1$ calmodulin-dependent protein kinase II at lateral amygdala synapses. J Neurosci 24: 3281-3288.

Rogan MT, LeDoux JE. 1995. LTP is accompanied by commensurate enhancement of auditory-evoked responses in a fear conditioning circuit. Neuron 15: 127-136.

Rogan MT, Staubli UV, LeDoux JE. 1997. Fear conditioning induces associative long-term potentiation in the amygdala. Nature 390: 604607.

Rosenkranz JA, Grace AA. 1999. Modulation of basolateral amygdala neuronal firing and afferent drive by dopamine receptor activation in vivo. J Neurosci 19: 11027-11039.

Rosenkranz JA, Grace AA. 2002a. Cellular mechanisms of infralimbic and prelimbic prefrontal cortical inhibition and dopaminergic modulation of basolateral amygdala neurons in vivo. J Neurosci 22 : 324-337.

Rosenkranz JA, Grace AA. 2002b. Dopamine-mediated modulation of odour-evoked amygdala potentials during Pavlovian conditioning. Nature 417: 282-287. 
Rosenkranz JA, Moore H, Grace AA. 2003. The prefrontal cortex regulates lateral amygdala neuronal plasticity and responses to previously conditioned stimuli. J Neurosci 23: 11054-11064.

Santini E, Ge H, Ren K, Pena de Ortiz S, Quirk GJ. 2004. Consolidation of fear extinction requires protein synthesis in the medial prefrontal cortex. J Neurosci 24: 5704-5710.

Santini E, Quirk GJ, Porter JT. 2008. Fear conditioning and extinction differentially modify the intrinsic excitability of infralimbic neurons. J Neurosci 28: 4028-4036.

Schafe GE, LeDoux JE. 2000. Memory consolidation of auditory Pavlovian fear conditioning requires protein synthesis and protein kinase $\mathrm{A}$ in the amygdala. J Neurosci 20: RC96-1-5.

Schafe GE, Atkins CM, Swank MW, Bauer EP, Sweatt JD, LeDoux JE. 2000. Activation of ERK/MAP kinase in the amygdala is required for memory consolidation of Pavlovian fear conditioning. J Neurosci 20: 8177-8187.

Schafe GE, Nader K, Blair HT, LeDoux JE. 2001. Memory consolidation of Pavlovian fear conditioning: A cellular and molecular perspective. Trends Neurosci 24: 540-546.

Sierra-Mercado D Jr, Corcoran KA, Lebron-Milad K, Quirk GJ. 2006. Inactivation of the ventromedial prefrontal cortex reduces expression of conditioned fear and impairs subsequent recall of extinction. Eur $J$ Neurosci 24: 1751-1758.

Sotres-Bayon F, Bush DE, LeDoux JE. 2007. Acquisition of fear extinction requires activation of NR2B-containing NMDA receptors in the lateral amygdala. Neuropsychopharmacology 32: 1929-1940.

Vidal-Gonzalez I, Vidal-Gonzalez B, Rauch SL, Quirk GJ. 2006.

Microstimulation reveals opposing influences of prelimbic and infralimbic cortex on the expression of conditioned fear. Learn Mem 13: 728-733.

Wan X, Puil E. 2002. Pentobarbital depressant effects are independent of GABA receptors in auditory thalamic neurons. J Neurophysiol 88: 30673077.

Weinberger NM, Gold PE, Sternberg DB. 1984. Epinephrine enables Pavlovian fear conditioning under anesthesia. Science 223: 605607.

Yaniv D, Schafe GE, LeDoux JE, Richter-Levin G. 2001. A gradient of plasticity in the amygdala revealed by cortical and subcortical stimulation, in vivo. Neuroscience 106: 613-620.

Received May 13, 2009; accepted in revised form October 23, 2009. 


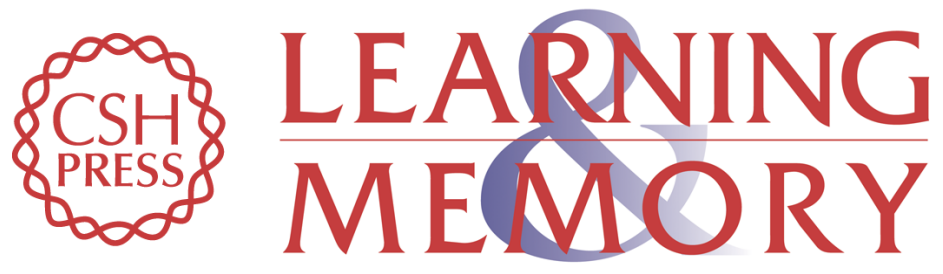

\section{Long-term synaptic changes in two input pathways into the lateral nucleus of the amygdala underlie fear extinction}

Junchol Park and June-Seek Choi

Learn. Mem. 2010, 17:

Access the most recent version at doi:10.1101//m.1482910

References This article cites 62 articles, 28 of which can be accessed free at:

http://learnmem.cshlp.org/content/17/1/23.full.htmI\#ref-list-1

License

Email Alerting Receive free email alerts when new articles cite this article - sign up in the box at the Service top right corner of the article or click here. 